\title{
Case Report \\ Severe Preeclampsia in the Setting of Myasthenia Gravis
}

\author{
Adam J. Lake, ${ }^{1}$ Antoun Al Khabbaz, ${ }^{1}$ and Renée Keeney ${ }^{2}$ \\ ${ }^{1}$ Department of Obstetrics and Gynecology, University of Illinois College of Medicine at Rockford, 1601 Parkview Ave., \\ Rockford, IL 61101, USA \\ ${ }^{2}$ Department of Women, Children, and Family Health, University of Illinois College of Nursing at Rockford, \\ 1601 Parkview Ave., Rockford, IL 61101, USA \\ Correspondence should be addressed to Antoun Al Khabbaz; akhabbaz@crusaderhealth.org
}

Received 29 October 2016; Accepted 17 January 2017; Published 9 February 2017

Academic Editor: Svein Rasmussen

Copyright (c) 2017 Adam J. Lake et al. This is an open access article distributed under the Creative Commons Attribution License, which permits unrestricted use, distribution, and reproduction in any medium, provided the original work is properly cited.

\begin{abstract}
Myasthenia gravis (MG) is a rare autoimmune disease that leads to progressive muscle weakness and is common during female reproductive years. The myasthenic mother and her newborn must be observed carefully, as complications during all stages of pregnancy and the puerperium may arise suddenly. Preeclampsia is a common obstetrical condition for which magnesium sulfate is used for seizure prophylaxis. However, magnesium sulfate is strongly contraindicated in MG as it impairs already slowed nervemuscle connections. Similarly, many first-line antihypertensive medications, including calcium channels blockers and $\beta$-blockers, may lead to MG exacerbation. This case describes the effective obstetrical management of a patient with MG who developed severe preeclampsia. The effective use of levetiracetam and various antihypertensive medications including intravenous labetalol is described. A review of the ten reported cases of MG complicated by preeclampsia is examined to aggregate observations of clinical care, with focus on delivery methods, anticonvulsants, and antihypertensive medications.
\end{abstract}

\section{Introduction}

Myasthenia gravis (MG) is an autoimmune disease in which antibodies most frequently target muscle nicotinic acetylcholine receptors (nAChR) or muscle-specific kinase (MuSK), which leads to the gradual attrition of neuromuscular signals. This manifests itself as fatigue and progressive paresis of skeletal muscle, which characteristically worsens with exertion and improves with rest. There is an estimated MG prevalence of 1 per 5,000 individuals in the United States [1] with maternal MG complicating 1 in 68,000 pregnancies [2]. Exacerbations of MG are termed myasthenic crises and are often precipitated by infections, antibiotics, emotional stress, and surgery [3]. A myasthenic crisis may lead to lifethreatening acute respiratory failure requiring mechanical ventilation. With improved neurocritical care protocols, mortality from a myasthenic crisis has improved to 5\% [3]. The Myasthenia Gravis Foundation of America (MGFA) treatment guidelines recommend the use of acetylcholinesterase inhibitors, intravenous immunoglobulins, plasma exchange (PLEX), glucocorticoids, and thymectomy for myasthenia treatment [4].

Preeclampsia is a systemic disorder characterized by newonset hypertension, proteinuria, and end-organ damage after 20 -week gestation and complicates $2-8 \%$ of pregnancies in the United States [5]. Treatment of hypertension in the setting of preeclampsia may be approached aggressively with multiple antihypertensive medications to achieve adequate blood pressure control. Magnesium sulfate, shown to be superior to other anticonvulsants, is used frequently for seizure prophylaxis [6]. Both MG and preeclampsia have specific treatment guidelines, which are often sufficient for adequate control of each disease. However, management of preeclampsia with magnesium sulfate and commonly used antihypertensive medications, such as $\beta$-blockers and calcium channel blockers, is contraindicated in MG as it may exacerbate MG symptomatology and precipitate a myasthenic crisis. An association between MG and preeclampsia prevalence has 
not been demonstrated in the English literature. There is a paucity of reports describing treatment of preeclampsia in patients with MG.

We present a pregnancy complicated by preexisting MG and the later development of severe preeclampsia with description of novel clinical management with intravenous levetiracetam and labetalol. A review of the English literature is presented as well, describing experiences with this rare clinical scenario.

\section{Presentation of Case}

A 28-year-old G3P2002 patient at 34-week gestation was admitted to the labor and delivery suite with a diagnosis of preeclampsia. The patient had two prior uncomplicated spontaneous vaginal deliveries. Her pregnancy was dated by ultrasound at 8 -week gestation. The patient is known to have MG managed with pyridostigmine $30.0 \mathrm{mg}$ orally, three times a day. She had a thymectomy six years prior to this pregnancy. She had an uncomplicated prenatal course. Her blood pressure during pregnancy ranged from 108 to $132 \mathrm{mmHg}$ systolic and 67 to $88 \mathrm{mmHg}$ diastolic. She has a history of preexisting prehypertension and was on no antihypertensive treatment. Upon arrival to the hospital, the patient's initial blood pressure was in the range of $170-180 \mathrm{mmHg}$ systolic and $100-110 \mathrm{mmHg}$ diastolic. She was in no acute distress and reported no clinically significant edema, right upper quadrant pain, weakness, dyspnea, diplopia, or ptosis. Her only notable symptom was a new-onset, mild headache. She denied experiencing contractions, leakage of fluid per vagina, or vaginal bleeding, and she reported normal fetal movements. Workup for preeclampsia showed an elevated protein/creatinine ratio of 0.7 , a slightly elevated uric acid of $5.6 \mathrm{mg} / \mathrm{dL}$, creatinine of $0.74 \mathrm{mg} / \mathrm{dL}$, normal liver enzymes (AST $26 \mathrm{U} / \mathrm{L}$, ALT $11 \mathrm{U} / \mathrm{L})$, and platelet count $152 \times 10^{3} / \mu \mathrm{L}$.

She was started on intravenous levetiracetam (1.0 g intravenous bolus for seizure prophylaxis). She was given multiple doses of $5.0 \mathrm{mg}$ intravenous hydralazine to treat her hypertension, which had minimal effect. Her blood pressure was as high as $229 / 117 \mathrm{mmHg}$. The patient was then given labetalol intravenously resulting in better control of her blood pressure with no MG exacerbation noted. Within a few hours of admission, the patient developed mild clonus and her headache increased in severity. The patient's cervix at this point was unfavorable so the decision was taken to proceed with Cesarean section under spinal anesthesia. Surgery was performed without complications, resulting in the birth of a healthy male newborn with a birth weight of $1865 \mathrm{~g}$ and APGAR scores of 8 at one minute and 9 at five minutes. The newborn was followed up closely and showed no signs of transient neonatal MG.

In the postpartum period, levetiracetam was continued at a dose of $500 \mathrm{mg}$ intravenously every 12 hours and was discontinued 2 days postpartum. The only notable side effects were somnolence and fatigue. These symptoms resolved after discontinuation of levetiracetam. Hypertension was treated with methyldopa and hydralazine. Clonidine was added on postpartum day 2. The patient was discharged home on postpartum day 4 on methyldopa and clonidine in stable condition with blood pressure in the prehypertensive range. The patient had no myasthenic crisis in either the intrapartum or the postpartum periods. She was weaned off her antihypertensive medications postpartum and was normotensive at her six-week postpartum visit.

\section{Discussion}

MG exacerbations are common in pregnancy, perhaps due to increases in blood volume and changes in the renal clearance of medications [17]. MG is associated with other autoimmune diseases such as rheumatoid arthritis and thyroid dysfunction but is not related to any increase in the prevalence of preeclampsia $[18,19]$. The clinical course of MG in pregnancy is variable, with an improvement of symptoms in $29 \%$ of pregnancies, worsening in 41\%, and no change in 30\% [20]. Pregnant patients with MG must be watched closely due to its unpredictable course in pregnancy. Chorioamnionitis, postpartum endometritis, and mastitis should be treated effectively as they can precipitate a myasthenic crisis [17]. Ideally, patients with MG should have optimization of their myasthenic symptoms prior to attempting to conceive [4]. Transient neonatal myasthenia gravis is due to transplacental passage of maternal IgG autoantibodies [17] and presents with mask-like facies, a weakened cry, and impaired ability to suck. Clinicians recommend neonatal observation for a minimum of 2 days prior to discharge $[4,21]$. Breastfeeding is not contraindicated in MG unless the patient is in a myasthenic crisis [15].

A comprehensive literature review of published cases of MG and preeclampsia in the English literature published from 1976 to 2016 using a MEDLINE search is presented (Table 1). Ten case reports have been published. A variety of medications have been implicated in inducing myasthenic crises and should be avoided in all MG patients (Table 2) $[17,22]$. Paradoxically, this includes the initiation of corticosteroid treatment, which causes exacerbations in $50 \%$ of patients [3]. These cases provide insight into the modes of delivery, anticonvulsants, and antihypertensive medications used for this clinical situation.

Delivery was accomplished by vaginal delivery in four of the reported cases; the other six patients delivered by Cesarean section. Surgical delivery may lead to exacerbations of MG and should only be performed for maternal or fetal indications [11]. For this case and previous cases, Cesarean sections were considered beneficial due to concerns of worsening maternal status from preeclampsia [12] with consideration taken that surgery itself could cause a myasthenic crisis. Regional anesthesia is preferred over general anesthesia for both vaginal and surgical deliveries. Of the reported cases, seven patients received regional anesthesia. One of those patients had profound vagal bradycardia following neuraxial anesthetic blockage, which required atropine and ephedrine to resolve [14]. General anesthesia with intubation was performed in only one case, and prolonged ventilation was needed postpartum. In that case, the muscle relaxant pancuronium was used. Muscle relaxants, both depolarizing and nondepolarizing, are also to be avoided as MG patients are quite sensitive to their effects [21]. 


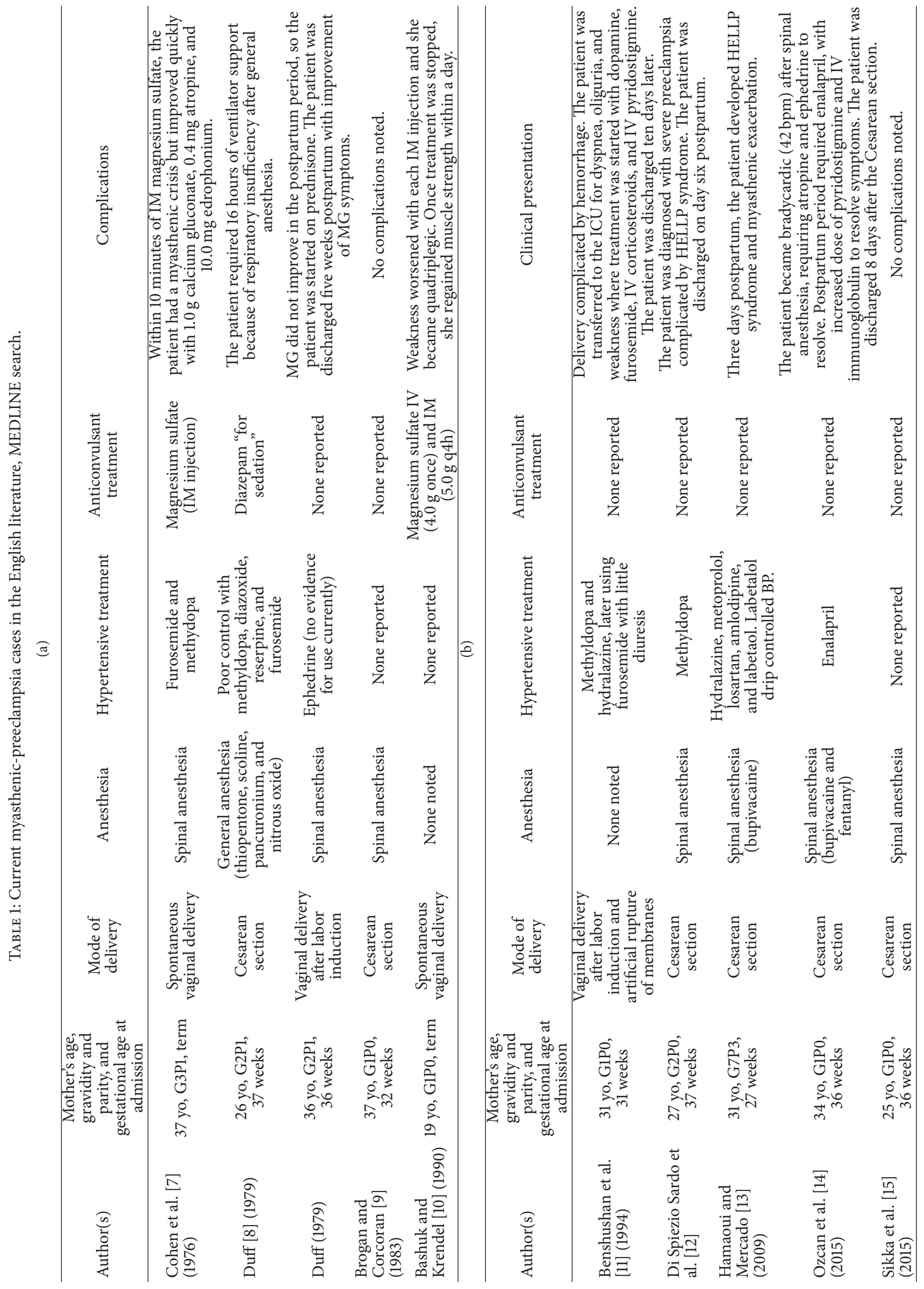


TABLE 2: Medications with associated exacerbations of myasthenia gravis.

\begin{tabular}{|c|c|}
\hline Magnesium salts & $\begin{array}{l}\text { Magnesium sulfate and milk of } \\
\text { magnesia, magnesium in multivitamins } \\
\text { acceptable }\end{array}$ \\
\hline $\begin{array}{l}\text { Calcium channel } \\
\text { blockers }\end{array}$ & Amlodipine, nifedipine, and verapamil \\
\hline$\beta$-Blockers & $\begin{array}{l}\text { Atenolol, propranolol, timolol } \\
\text { (including eye drops), and nadolol }\end{array}$ \\
\hline Aminoglycosides & $\begin{array}{l}\text { Gentamycin, clindamycin, } \\
\text { streptomycin, and to lesser extent } \\
\text { tobramycin }\end{array}$ \\
\hline Macrolides & Azithromycin and erythromycin \\
\hline Fluoroquinolones & Ciprofloxacin and levofloxacin \\
\hline D-Penicillamine & $\begin{array}{l}\text { Strong association with worsening } \\
\text { symptoms of myasthenia, considered } \\
\text { contraindicated }\end{array}$ \\
\hline Psychiatric medications & TCAs, haloperidol, and lithium \\
\hline Steroids & $\begin{array}{l}\text { Often used in myasthenia treatment, } \\
\text { causing transient worsening of } \\
\text { symptoms }\end{array}$ \\
\hline Statins $[16]$ & $\begin{array}{l}\text { Atorvastatin, simvastatin, rosuvastatin, } \\
\text { lovastatin, and pravastatin }\end{array}$ \\
\hline
\end{tabular}

Our patient received spinal anesthesia for her Cesarean section due to concerns of worsening severe preeclampsia. The surgery was uncomplicated and the patient had no residual side effects from the anesthesia.

Calcium channel blockers and $\beta$-blockers can exacerbate MG symptoms [14]. Hence, their use should be avoided if possible for a patient with MG. Methyldopa and hydralazine are considered safe to use in MG patients. Severe hypertension $(\geq 160 / 110 \mathrm{mmHg}$ ) should be treated aggressively to prevent stroke and intracranial bleeding. Intravenous labetalol is widely used for treatment of severe hypertension in the intrapartum and postpartum periods. However, its use can cause weakness in MG patients. As such, patients should be monitored closely due to potential worsening of weakness [14]. A labetalol intravenous drip was effective in controlling severe hypertension in one reported patient with MG and preeclampsia [13]. The patient had transient weakness without any other significant neurologic side effects. Labetalol use should be restricted to cases with poor blood pressure control with hydralazine, and close monitoring of MG symptoms should be implemented.

Our patient was treated first with multiple doses of $5.0 \mathrm{mg}$ intravenous hydralazine but her blood pressure remained as high as $229 / 117 \mathrm{mmHg}$. Labetalol $20.0 \mathrm{mg}$ intravenously was given, leading to adequate blood pressure control with no worsening fatigue, weakness, or other symptoms of MG. A total of three doses of labetalol intravenously were given.

The American Congress of Obstetrics and Gynecology recommends the use of magnesium sulfate for seizure prophylaxis in preeclampsia; however, magnesium sulfate is strongly contraindicated in MG as it impairs already slowed nerve-muscle contractions through a competitive mechanism involving calcium at the neuromuscular junction [23]. Serum magnesium concentrations cause typical, dose-dependent side effects such as fatigue, facial flushing, arrhythmias, and muscle weakness. In neuromuscular disease, symptoms develop at lower magnesium levels [9]. The first reported case of MG with preeclampsia was treated with two intramuscular doses of $5.0 \mathrm{~g}$ of magnesium sulfate [6]. The patient developed weakness and profound obtundation followed by sudden acute respiratory insufficiency. Of the ten previously reported cases, anticonvulsant treatment has been reported in three cases. Two patients were treated with magnesium sulfate and one patient was treated with diazepam. Both patients treated with magnesium sulfate had a myasthenic crisis; one of them developed transient quadriplegia $[7,8,10]$.

Few studies suggest phenytoin and diazepam as an alternative for seizure prophylaxis in preeclampsia. Systematic reviews concluded that they are less effective than magnesium sulfate in reducing mortality $[18,24]$. A UK multispecialty group recommended magnesium sulfate use only in those women who experience eclamptic seizures, with the caveat that mechanical ventilation should be prepared and an obstetrical anesthesiologist and neurologist should be on site [21].

Our patient was treated with levetiracetam for seizure prophylaxis. The patient did well with no significant neurological symptoms, eclamptic seizures, or myasthenic symptoms. Levetiracetam is a relatively safe (Pregnancy Risk Category C) antiepileptic medication. Advantages for use in pregnancy include a lower need for drug level monitoring and fewer interactions with other medications. A recent systematic review showed no significant increase in major congenital malformations compared with unexposed controls [25].

Obstetrical care of the myasthenic patient is ideally a team-based approach involving obstetricians, neurologists, anesthesiologists, and neonatologists. The pregnant patient with MG and preeclampsia should be hospitalized with any sign of elevated blood pressure. Cesarean section with spinal anesthesia should be performed only for obstetrical indications as it may precipitate a myasthenic crisis but has generally good outcomes. Hydralazine is considered the drug of choice for treatment of severe preeclampsia, while intravenous labetalol can be used for refractory hypertension but requires close monitoring of the patient. Levetiracetam can potentially be used effectively and safely for seizure prophylaxis in this rare clinical scenario.

\section{Ethical Approval}

This project was reviewed and approved by the University of Illinois College of Medicine IRB, Project ID no.: 876654-1.

\section{Consent}

Written permission for publication was obtained from the patient.

\section{Competing Interests}

The authors declare that there are no competing interests regarding this publication. 


\section{References}

[1] L. H. Phillips II, "The epidemiology of myasthenia gravis," Annals of the New York Academy of Sciences, vol. 998, pp. 407412, 2003.

[2] F. F. Schade and M. P. Foley, "Pregnancy in myasthenia gravis," American Journal of Obstetrics and Gynecology, vol. 63, no. 5, pp. 1154-1156, 1952.

[3] D. A. Godoy, L. J. V. de Mello, L. Masotti, and M. Di Napoli, "The myasthenic patient in crisis: an update of the management in Neurointensive care unit," Arquivos de Neuro-Psiquiatria, vol. 71, no. 9 A, pp. 627-639, 2013.

[4] D. B. Sanders, G. I. Wolfe, M. Benatar et al., "International consensus guidance for management of myasthenia gravis: executive summary," Neurology, vol. 87, no. 4, pp. 419-425, 2016.

[5] L. Duley, "The global impact of pre-eclampsia and eclampsia," Seminars in Perinatology, vol. 33, no. 3, pp. 130-137, 2009.

[6] L. Duley, A. M. Gülmezoglu, D. J. Henderson-Smart et al., "Magnesium sulphate and other anticonvulsants for women with pre-eclampsia," The Cochrane Database of Systematic Reviews, no. 11, Article ID CD000025, 2010.

[7] B. A. Cohen, R. S. London, and P. J. Goldstein, "Myasthenia gravis and preeclampsia," Obstetrics and Gynecology, vol. 48, supplement 1, pp. 35S-37S, 1976.

[8] C. B. Duff, "Preeclampsia and the patient with myasthenia gravis," Obstetrics and Gynecology, vol. 54, no. 3, pp. 355-358, 1979.

[9] M. Brogan and D. J. D. Corcoran, "Myasthenia gravis and preeclampsia," Irish Medical Journal, vol. 76, no. 2, pp. 84-85, 1983.

[10] R. G. Bashuk and D. A. Krendel, "Myasthenia gravis presenting as weakness after magnesium administration," Muscle and Nerve, vol. 13, no. 8, pp. 708-712, 1990.

[11] A. Benshushan, N. Rojansky, and D. Weinstein, "Myasthenia gravis and preeclampsia," Israel Journal of Medical Sciences, vol. 30, no. 3, pp. 229-233, 1994.

[12] A. Di Spiezio Sardo, A. Taylor, M. Pellicano et al., "Myasthenia and HELLP syndrome," European Journal of Obstetrics Gynecology and Reproductive Biology, vol. 116, no. 1, pp. 108-111, 2004.

[13] A. Hamaoui and R. Mercado, "Association of preeclampsia and myasthenia: a case report," Journal of Reproductive Medicine, vol. 54, no. 9, pp. 587-590, 2009.

[14] J. Ozcan, I. F. Balson, and A. T. Dennis, "New diagnosis myasthenia gravis and preeclampsia in late pregnancy," $B M J$ Case Reports, vol. 2015, Article ID e208323, 2015.

[15] P. Sikka, B. Joshi, N. Aggarwal, V. Suri, and H. Bhagat, "Distinguishing myasthenia exacerbation from severe preeclampsia: a diagnostic and therapeutic challenge," Journal of Clinical and Diagnostic Research, vol. 9, no. 8, pp. QD05-QD06, 2015.

[16] J. Gale and H. V. Danesh-Meyer, "Statins can induce myasthenia gravis," Journal of Clinical Neuroscience, vol. 21, no. 2, pp. 195197, 2014.

[17] M. Varner, "Myasthenia gravis and pregnancy," Clinical Obstetrics and Gynecology, vol. 56, no. 2, pp. 372-381, 2013.

[18] W. C. Plauche, "Myasthenia gravis in pregnancy," American Journal of Obstetrics and Gynecology, vol. 88, pp. 404-409, 1964.

[19] B. Piura, "The association of preeclampsia and myasthenia gravis: double trouble," Israel Journal of Medical Sciences, vol. 30, no. 3, pp. 243-244, 1994.

[20] A. P. Batocchi, L. Majolini, A. Evoli, M. M. Lino, C. Minisci, and P. Tonali, "Course and treatment of myasthenia gravis during pregnancy," Neurology, vol. 52, no. 3, pp. 447-452, 1999.
[21] F. Norwood, M. Dhanjal, M. Hill et al., "Myasthenia in pregnancy: best practice guidelines from a UK multispecialty working group," Journal of Neurology, Neurosurgery and Psychiatry, vol. 85, no. 5, pp. 538-543, 2014.

[22] A. Elsais, T. H. Popperud, Ø. Melien, and E. Kerty, "Medikamenter som kan utløse og forverre myasthenia gravis," Tidsskrift for Den Norske Legeforening, vol. 133, no. 3, pp. 296-299, 2013 (Norwegian).

[23] D. H. Jenkinson, "The nature of the antagonism between calcium and magnesium ions at the neuromuscular junction," The Journal of physiology, vol. 138, no. 3, pp. 434-444, 1957.

[24] J. N. Mueksch and W. A. Stevens, "Undiagnosed myasthenia gravis masquerading as eclampsia," International Journal of Obstetric Anesthesia, vol. 16, no. 4, pp. 379-382, 2007.

[25] S. A. Chaudhry, G. Jong, and G. Koren, "The fetal safety of Levetiracetam: a systematic review," Reproductive Toxicology, vol. 46, pp. 40-45, 2014. 


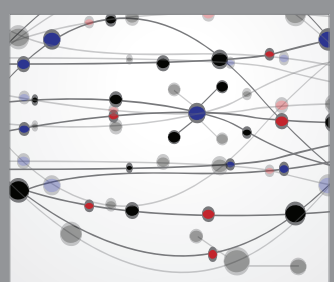

The Scientific World Journal
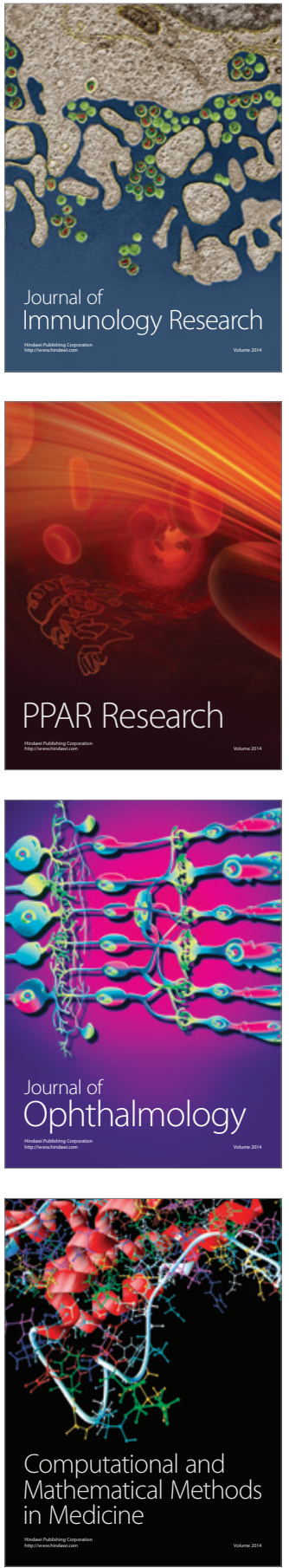

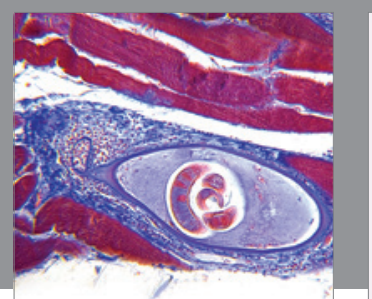

Gastroenterology Research and Practice
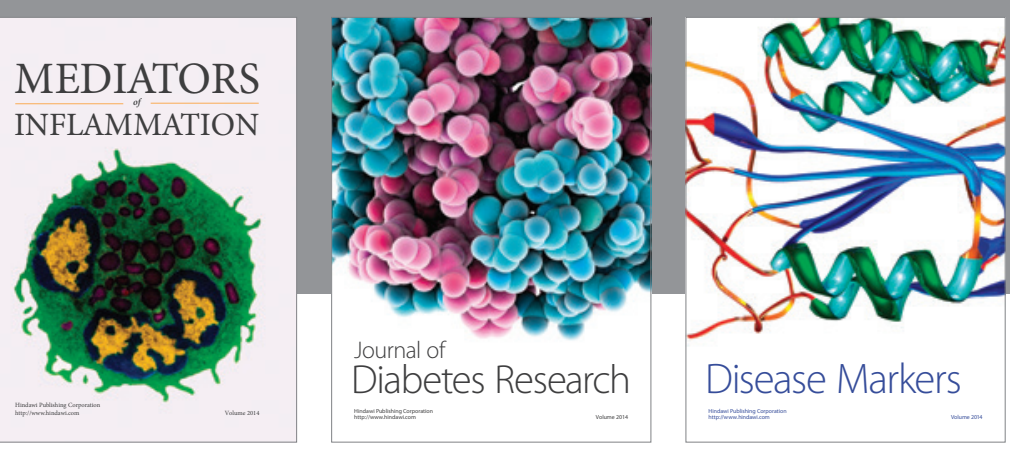

Disease Markers

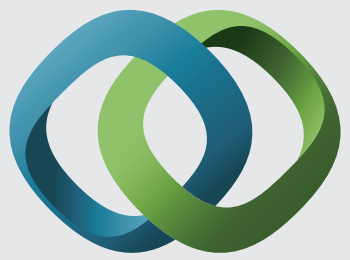

\section{Hindawi}

Submit your manuscripts at

https://www.hindawi.com
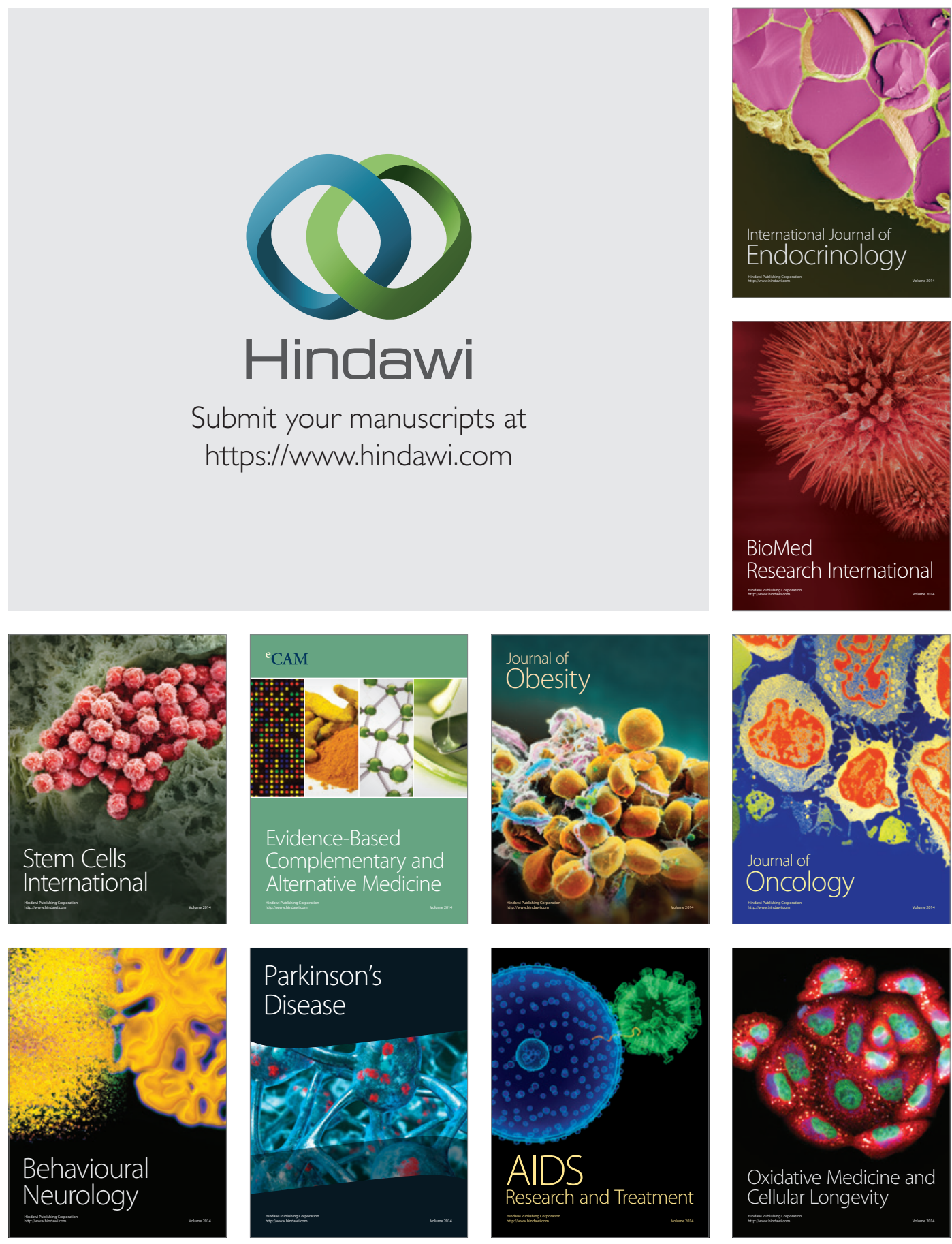